\title{
A Historical Review of Robert van Gulik and His Judge Dee Mysteries in Chinese World (1996-2016)*
}

\author{
WANG Jin, MO Wan-yi \\ Faculty of Foreign Studies, Jinan University, Guangzhou, PR.China
}

\begin{abstract}
Judge Dee Mysteries was written by a Dutch diplomat and orientalist Robert van Gulik, and has been translated into more than twenty languages, gaining a wide attention with the honorary title of "Oriental Sherlock Holmes" in English world. It returned to China with Chen Laiyuan and Hu Ming's translations in 1980s, and since then there emerged various studies by Chinese scholars on Robert van Gulik and his works. This article makes a historical review of Judge Dee Mysteries in Chinese world during the past 20 years, presents problems in translating and introducing Robert van Gulik in Chinese, and proposes comments and suggestions for further translation and studies of Judge Dee Mysteries in a comparison with Chinese gong'an fictions.
\end{abstract}

Keywords: Robert van Gulik, Judge Dee Mysteries, Chinese gong’an fictions, translation studies

\section{Introduction}

Robert van Gulik (1910-1967), a famous Dutch diplomat and sinologist, is the author of Judge Dee Mysteries. He is also an expert in many areas of Chinese culture including the Guqin (a Chinese musical instrument), sexology, calligraphy and etc. Judge Dee Mysteries, the series of gong'an fiction (a genre similar to Western detective stories), rooted in procedural criminal laws of ancient China, writes about a magistrate judge named Judge Dee in Tang Dynasty. Historically speaking, the collection of stories has been patterned after a real jurist-statesman named Di Renjie in Tang Dynasty, solving different (and sometimes unrelated) murder cases in each book with the help of three assistants. The writer follows the long tradition of Chinese gong'an fiction and preserves a number of key elements of Chinese culture of gong'an fiction. With 16 novels and 8 short stories, Judge Dee Mysteries prove to be a great success in introducing Chinese literature and culture to Western readers in English world.

Van Gulik's original intention in writing his first Judge Dee novel was, as he wrote in remarks on The Chinese Bell Murders, "to show modern Chinese and Japanese writers that their own ancient crime-literature has plenty of source material for detective and mystery-stories”(1977, p. ii). He made a great effort to arouse modern Chinese's attention on recognizing their own gong'an stories. Besides, he intended to efface Western stereotypes

\footnotetext{
* Acknowledgements: The paper is supported by the Fundamental Research Funds for the Central Universities (Code: 15JNQM021) and the Research Funds for Outstanding Young University Teachers of Guangdong Province (Code: 2014-108-31). WANG Jin (the correspondent author) is currently an Associate Professor of English literature at the Faculty of Foreign Studies, Jinan University, and he is mainly interested literary studies and cultural studies. MO Wan-yi is an M.A. Student of translation studies and she is mainly interested in translation and translation studies.
} 
and prejudices on China and their crime literature, educating Western readers with an authentic knowledge of a real China. Chinese scholar Zhao Yiheng is the first one to introduce Judge Dee Mysteries to Mainland China, highly appreciating van Gulik and his Judge Dee Mysteries. As he points out,

The greatest contributions to spreading Chinese culture were made, in a sense, probably by van Gulik. The influences of his Judge Dee mysteries far exceed those of any works about Chinese studies. For many Westerners in that period, impressions and understanding of China were mostly from the Judge Dee mysteries, which were popular with the Western readers. (ZHAO, 2002)

When most Westerners have negative impressions and prejudices on China,

Van Gulik tried to efface the demonized oriental impressions in the Westerner's minds with his Chinese studies and crime literature, which has successfully corrected part of their opinions on China and helped to build a positive and fair image especially for China. (ZHANG, 2010, p. 12)

This paper presents a historical review of the recent studies on the Judge Dee Mysteries in the following five aspects, providing a full picture of its findings and problems and exploring possibilities for further studies of van Gulik and his Judge Dee Mysteries.

\section{Recent Studies of van Gulik and His Judge Dee Mysteries}

In recent years there is a significant growth of scholars engaged in the studies of van Gulik, most of whom are only limited to different versions of biographical introductions of van Gulik, and there are few monographs with a focused topic or a systematic argument published. This situation may come from mainly the following three problems. First, the studies by Chinese scholars started only from the 1980s when van Gulik and his series of stories were translated by Chen Laiyuan and Hu Ming into Chinese, and it was relatively late for academic studies. Related research literature (especially in Chinese) about van Gulik and his works are relatively limited in both numbers and depth, making it difficult for Chinese scholars with few references to conduct further studies. Second, as detective fiction is one of branch of popular fictions, "the studies of Chinese detective fiction are not abundant either in breadth or depth” (ZHANG, 2010, p. 32), compared with the Western ones. Many Chinese scholars had not paid enough attention to study detective fictions that they overlooked Judge Dee Mysteries and this kind of fictions. Third, as the series of stories preserve key elements of Chinese culture, further studies may require researchers' good command of both Chinese and English languages, along with a profound knowledge of both cultures.

Wilt L. Idema, a professor of Chinese Literature at Harvard University, has been influential for his studies of van Gulik and his Judge Dee Mysteries in Chinese world. He began to study van Gulik in 1970s, whose articles have been translated, published and quoted for many times in Mainland China. His paper "Dutch Sinology: Past, Present and Future" gives a brief introduction to van Gulik's sinology and his Judge Dee Mysteries and he highly appreciates his status and legacies in Dutch sinology (79-86). Donald F. Lach, a history professor at the University of Chicago, is also a notable one in studying van Gulik. In the late 1970s, he wrote a long preface for Judge Dee Mysteries published by the University of Chicago, in which he introduced the whole story of van Gulik's writing of Judge Dee Mysteries, analyzed the true Chinese images and the imaginary ones shown in the books, spoke highly of the writing techniques that were more adapted to a Western audience. After all, out of a 
limited knowledge of Chinese language and culture, the research on van Gulik and his Judge Dee Mysteries conducted by scholars outside China does not flourish.

The domestic research on van Gulik starts relatively late. It emerged only in the 1980s when the series of gong'an novels were translated. By the end of October, 2016, there have been nearly 50 papers on the studies of Judge Dee Mysteries published by Chinese journals (Indexed in the database of China National Knowledge Infrastructure). From 1980s to the early 20th century, most of the studies of van Gulik and its works are limited to a brief introduction of van Gulik's life, his sinology and Judge Dee Mysteries. Among them, the most representative papers are Hou Min’s “Robert van Gulik and the Judge Dee Mysteries”, Zhao Yiheng’s “Famous Scholar van Gulik and His Judge Dee Mysteries" and "One-century van Gulik, 60-year Di Renjie”, Chen Laiyuan’s “van Gulik and His Judge Dee Mysteries” and Zhao Ke’s “Dutch Sinologist Robert van Gulik Making Detective Di Renjie Widely Known” and so on. Since the year of 2006, and especially after 2009, more and more domestic scholars realize the significance of van Gulik and actively participate in the studies of van Gulik and his Judge Dee Mysteries. They attempt to fill the blind spots in the studies of van Gulik from different perspectives. This paper provides a historical review of the studies of Judge Dee Mysteries and locates them mainly into the following five different categories.

\section{Improvement and Development of Gong'an Fiction in Judge Dee Mysteries}

Zuo Menglin and Hu Qin, in their co-authored paper “Van Gulik’s Judge Dee Mysteries’ Improvement and Development from Traditional Chinese Gong' an Fiction”, make a comparative study between the differences of traditional Chinese crime fiction and the Western one. They discuss improvements and developments made in Judge Dee Mysteries by employing the writing techniques of the Western detective fiction in three aspects such as structure modes of solving crime cases, narrative mode and plot arrangement. And they believes van Gulik not only contributes to injecting new vitality for the traditional Chinese gong' an fiction, but also provides possible ways for future's literature writing and innovation (ZUO \& HU, 2015, pp. 69-73).

In his paper "Case-solving and Detective Novels: Starting from Dee Gong An”, Wei Quan makes an analysis about connections and differences between 18th-century gong'an novel Dee Gong An and van Gulik's Judge Dee Mysteries, and then explains possible reasons for the differences between traditional Chinese gong'an fiction and the Western one. Besides, he also makes a comparative study of detective novels written by Wu Jianren and Cheng Xiaoqing with those by van Gulik, drawing a conclusion that a perfect combination of "shell of traditional Chinese gong'an fiction” and “modern Western thought and ideas” enables Judge Dee Mysteries to present their distinctive artistic style (Wei, 2006, p. 38). Besides, Guo Mengying's paper "The Judge Dee Mysteries' Creative Continuation and Development from Chinese Gong'an Fiction” shares the same research topic (GUO, 2014, pp. 88-89). Although his paper does provide a new perspective for literature with the writing techniques of combining the oriental and Western elements in Judge Dee Mysteries, they are also limited to a relatively narrow perspective. As a matter of fact, there are no attempts of any kind to explore reasons for such creative continuation and development of traditional Chinese gong'an fiction.

\section{Cross-cultural Adaptations in Judge Dee Mysteries}

In his paper “Circulation Brings Magic—Huo-sang’s Detective Stories vs The Judge Dee Mysteries”, Tang 
Zhesheng argues that Huo-sang's Detective Stories written by Cheng Xiaoqing (a Chinese detective story writer) tends to be more westernized while Judge Dee Mysteries closer to traditional Chinese gong'an fiction. He applies an analytical framework of "adaptation" and "misinterpretation" in the cross-cultural context, and believes that Cheng Xiaoqing is unconscious of "adaptation” and “misinterpretation” shown in Huo-sang's Detective Stories while van Gulik, with a good command of both Chinese and English languages and cultures, is more qualified by consciously mixing the elements of Western detective stories with those of traditional Chinese gong'an fiction. Tang speaks highly of the necessity of "adaptation” and "misinterpretation” (2009, pp. 93-97), focusing on reasons for the differences in the aspect of cross-cultural communication.

Shi Ye's paper "West-transmission and East-return of Chinese Court-case Novels under Intercultural Context” expounds the ways in which The Chinese Maze Murders is restructured as a traditional Chinese gong' an fiction to reach Western readers and how the novel returns to its original readers from the aspects of specific way of story-telling and cultural return as well as latency pattern maintenance in the cross-cultural communication.

Her paper examines the differences between van Gulik's self-translation version and Chen's version, and concludes that van Gulik's creation of Judge Dee successfully lead to readers' different imagination and interpretation, making the novel a successful case for the transmission of traditional Chinese gong'an fiction to the West and its return to the East. She makes efforts to prove that, whether it is the original English version, van Gulik's self-translation version or Chen's translation version, all adaptation or changes are made based on different times, cultures, social needs or value to give Judge Dee an entirely different interpretation (Shi, 2011, pp. 167-176). On the other hand, Wei Yan, in her paper "Cross-cultural Circulation of Judge Dee Stories", examines the courses of "traveling” of the particular genre from traditional Chinese gong'an fiction to Western detective one, and applies the concept of déjà vu to interpret why van Gulik’s Judge Dee Mysteries enable different readers to produce both exotic and nostalgic feelings (Wei, 2009, pp. 80-92).

\section{Translation Studies of van Gulik and His Judge Dee Mysteries}

The above mentioned papers examine possible reasons for changes and adaptation of van Gulik's works, but they seldom highlight specific translation methods and strategies involved. As a matter of fact, it is of great value to study van Gulik and his Judge Dee Mysteries in an analytical framework of translation studies. For example, Ge Wei completed in her master's thesis “A Brief Discussion between The Chinese Nail Murders and Tie Ding $A n$ ”, and argued that Chen Laiyuan and Hu Ming have adopted a translation strategy of domestication, deleting the content of Chinese folk culture, subplots and psychological description, rewriting some plots, and changing the narrative mode and writing style, only to meet Chinese readers' expectation and the needs of Chinese society. In her paper “Dynamic Equivalence Theory and Domestication in the Judge Dee Mysteries' Chinese Version”, $\mathrm{Xu}$ Taoping also points out Chen and Hu's adoption of domestication in cultural background, value orientation, narrative mode, and language style, with an eye toward target readers, whose translation embodies the application of Nida's dynamic equivalence theory.

Besides, Lin Junhong's MA thesis “Tracking down the Old China: Focusing on the Translation from The Chinese Maze Murders”, applies the rewriting theory of Andre Lefevere, and examines the same research topic, making a comparative study between English original version of The Chinese Maze Murders and its three Chinese versions respectively by van Gulik himself, Chen and Taiwan Lianpu publisher. In his thesis, Lin mainly 
explores reasons for different ideologies behind the image of "traditional China" from different Chinese versions. Another scholar Huang Haiyan also employs Andre Lefevere's rewriting theory to compare van Gulik' s Judge Dee Mysteries with Chen's Chinese version in her paper "Plot Changing in the Chinese Version of The Judge Dee Mysteries”. She argues that ideology, poetics and sponsor are the three elements influencing the translators to make adaptations and concludes that the great success of Chen's version has much to do with the application of the translation strategy of rewriting.

Different from the rewriting approach, Zhang Yan employs Qian Zhongshu's "E” (means “error”) translation thought in her master's thesis "A Study on the Phenomenon of "E” in Da Tang Di Gong An", and makes a systematic analysis on the " $E$ " phenomenon in the linguistic level, textual level and cultural level with a large number of typical examples selected. In conclusion, she recognizes the feasibility and necessity of "E" translation thought embodied in Chen's translation version, and points out that this phenomenon is influenced by the translators' own aesthetic experiences, Chinese traditional ideology and mainstream poetics. Another Chinese scholar Chen Cuiqin, in her paper "Studies on Chinese Version of van Gulik's The Emperor's Pearl”, employs Skopo theory to analyze the "Second Transformation" in the process of the novel being translated back into Chinese. In "Translating Chinese Gong’an Fiction in the Light of Narrative Mode”, Wang Baorong and Yao Wei believe that van Gulik's Judge Dee Mysteries is a good representative for "special translation” of traditional Chinese gong'an fiction and it lays a particular emphasis on the adaptation of the narrative mode in the series of gong'an novel and creative rewriting based on the traditional Chinese fiction. The paper draws a conclusion that the westernization of narrative mode in the translation process can help Chinese gong'an fiction to walk toward the world.

In this regard, Wang Hongyin conducts translation studies on Judge Dee Mysteries in his paper "Towards a Universal Theory of Translation Text-less Back Translation Reviewed with Examples from Judge Dee Mystery and Other Works". He argues that Judge Dee Mysteries and its Chinese translation version contribute to promoting the completeness of the basic theory, including concepts of non-native language writing and text-less back translation. The paper also reviews a number of disciplines and typology of terminologies in an attempt to justify his theory both theoretically and practically with examples from Judge Dee Mysteries. The author at the end of his argument expresses his personal hope that text-less back translation can be a useful translation concept to promote Chinese classics as well as modern literary works to go global.

\section{Traditional Images of China in Judge Dee Mysteries}

Traditional image of China in Judge Dee Mysteries were not always authentic as some of them were imagined by van Gulik either consciously or unconsciously. Wang Wenjuan discusses traditional image of China from the perspective of imagology in her master's thesis "Between Ideological and Utopian Extremes—Chinese Image in Robert van Gulik’s Judge Dee Mysteries”. She first of all presents a related background of image analysis in the field of comparative literature, and then analyzes words, hierarchical relationships and plots of the novel through internal-text research, sorting out Chinese images in three aspects, namely Chinese administration of the officials, the bisexual relationships and folk beliefs through external research. Her thesis concludes that Chinese images in Judge Dee Mysteries are neither too ideological nor utopian, but always in appropriate negotiations between the two. 
In her master thesis "Image of China in Robert van Gulik’s Judge Dee Mysteries”, Rong Xia also adopts a perspective of imagology to explore the ways in which van Gulik produced images of China and the relationships between images and social factors. Besides, there are papers discussing the images of certain characters in Judge Dee Mysteries. For example, Wang Yan’ paper “Female Prisoners in the Judge Dee Mysteries” discusses van Gulik's misinterpretation of ancient Chinese society by analyzing images of female prisoners in Judge Dee Mysteries and 18th-century gong'an novel Dee Goong An. Wang believes that it is because van Gulik was deeply subjected to Western values that he misinterpreted ancient Chinese female characters. However, as Wang's paper concludes, even though van Gulik gives a factual account of the differences of the image of female prisoners, his misinterpretation of traditional Chinese culture was consciously "designed”. However, Zhang Ping argues against this point and she emphasizes that "the female images that van Gulik molded is a breakthrough, which is added some Western feminist elements to show a perfect combination of Chinese and Western echoes” (ZHANG, 2010, p. 150). In this regard, the paper "Combination of Virtue and Wisdom—Image of Judge Dee from van Gulik”, written by Zuo Menglin and Hu Qin, argues with specific examples that Judge Dee Mysteries combines quality of "worship of virtue" in traditional Chinese gong' an fiction with that of "worship of wisdom" in Western detective stories, representing images of virtual and wise Judge Dee.

\section{Examples of Comprehensive Studies of Judge Dee Mysteries}

In addition to the papers centered on one certain subject, there are also papers that cover more than one subject, indicating an increasing critical attention to van Gulik and his Judge Dee Mysteries, particularly in the field of cross-cultural communication and its specific strategies. For example, Deng Chu, in her master's thesis “A Study on Robert van Gulik’s Judge Dee Mysteries”, discusses the integration of Chinese and the Western elements, focusing on images of protagonists and female characters, issues of law and justice, and in this way speaks highly of the value of van Gulik's strategies to promote cross-cultural transmission.

In her "Cultural Studies on Robert van Gulik and His Judge Dee Mysteries”, Zhang Ping also makes a comprehensive study on van Gulik and his Judge Dee Mysteries as early as 2007. It examines strategies of cultural translations in Chen Laiyuan's translation, and has a detailed analysis of its narrative structures, character portraying and different values between China and the West. Quoting a large number of first-hand sources and materials, Zhang provides her insightful and convincing views and evaluation of van Gulik and his Judge Dee Mysteries.

From a perspective of cultural studies, Luo Hailan's doctoral dissertation "A Comparative Study of Sino-West Culture Involving in Detective Novels Starting from Robert van Gulik’s 'The Judge Dee Mysteries’” adopts the series as a model to study cultural values and exchanges, especially with a conceptual framework of cultural exports and their strategies. Besides, he also takes the series as a reference text, and makes an in-depth discussion of differences between Chinese and Western legal cultures. His research contributes to opening new space for text studies and broadening the horizon of doing studies on the Judge Dee mysteries, in consideration of the development of Judge Dee Mysteries in future TV plays, along with a common pursuit of social equality and justice in the heterogeneous cultures between China and the West. 


\section{Further Studies on van Gulik and His Judge Dee Mysteries}

For future studies on van Gulik and his Judge Dee Mysteries, his detective novels and sinology will receive more and more attention in a global context, as cross-cultural exchanges become more and more frequent and there are increasing needs for Chinese literary works to "go abroad". As a Dutch using English to write Chinese detective stories, van Gulik can be regarded as a special cultural translator to introduce Westerners traditional Chinese culture by producing his series of gong'an fiction. Van Gulik's works would inevitably contain certain Western characteristics and traces, thereby the translation studies on culture back translation and culture restoration would unavoidably occur. But, in the current literature, translation study of van Gulik and his Judge Dee Mysteries involving back translation is relatively rare. In addition, any further studies of van Gulik and his Judge Dee Mysteries will cover at least the following three fields: (a) comparative translation studies on van Gulik's Judge Dee Mysteries and their Chinese translation version by Chen and Hu, among which The Chinese Maze Murders, the only novel translated by van Gulik himself will be studied at a full length; (b) wide range of studies on many aspects of traditional Chinese culture reflected in Judge Dee Mysteries, focusing more on political and economic culture, legal culture, traditional festivals, folk customs and moral ethics; (c) studies on how van Gulik's sinology interacts with his Judge Dee Mysteries. As the studies of van Gulik and his works go further within an interdisciplinary rationale, its cultural implications, historical significance, as well as theoretical spaces will be fully re/interpreted in a historic-cultural transformation of Sino-Western relations.

\section{Conclusion}

As one of the most important Dutch sinologist, Robert van Gulik and his Judge Dee Mysteries not only introduces Chinese gong' an fictions and its liteary signigicance into the Western world, but also enriches the Western literature with ancient Chinese history and cutlure of judical insitutions. This paper presents a historical overview of Robert van Gulik and his Judge Dee Mysteries translated and studied in contemproary Chinese world during the very pierod from 1996 to 2016, and classfies the recent literarue of van Gulik studies in accordance with five different thematic frameworks. Based on such a categorization, it further examines the study of of van Gulik and his Judge Dee Mysteries in such fields of cultural studies of translation, genre studies of gong'an fiction, cross-culutral adaptations, traditional Chinese images, comprehensive studies of ancient Chinese society. By presenting a whole picture of the achievenments and limitations of van Gulik studies, the paper aruges that any furhter exploration should first all historicizes van Gulik as a Dutch sinogloist and provides a contextualized study of van Gulik's works in both English and Chinese worlds. With the growing importance of sinology or Chinese studies in today's China, the translation and in this way transltion studies of van Gulik re-introduces Western interpretations of Chinese cutlure back into the Chinese wolrd, and provides an alternative way for Chinese critics to reinterpret or reappreicate their own language and culture. In this way, the author belives that the historical signifiance of van Gulik lies not only in current studies of his enthusiasatic reinovations of the traditional literary genre of Chinese gong'an fiction, but also in future explorations of his deliberate promotions of Chinese cultural values in Chinese literary classics.

\section{References}

Chen, C. Q. (2004). Studies on Chinese version of van Gulik's The Emperor's Pearl. Taiwan: Fu Jen Catholic University. 
GUO, M. Y. (2014). The Judge Dee Mysteries’ creative continuation and development from Chinese Gong'an fiction. Masterpieces Review: Literature Studies, (2), 88-91.

HUANG, H. Y. (2015). Plot changing in the Chinese version of The Judge Dee Mysteries. Journal of Harbin University, (7), 95-100.

Idema, W. L. (1993). Dutch sinology: Past, present and future. (Q. H. MA and W. TANG, Trans.). Traditional Culture and Modernization, (1), 79-86.

LIN, J. H. (2007). Tracking down the Old China: Focusing on the translation from The Chinese Maze Murders. Taiwan: National Taiwan Normal University.

SHI, Y. (2011). West-transmission and East-return of Chinese court-case novels under intercultural context. Journal of Social Sciences, (6), 167-176.

TANG, Z. S. (2009). Circulation brings magic-Huo-sang's Detective Stories vs The Judge Dee Mysteries. Journal of Jianghan Luntan, (5), 93-97.

Van Gulik, R. (1977). The Chinese bell murder. Chicago: University of Chicago Press.

WANG, B. R., \& YAO, W. (2014). In translating Chinese Gong'an fiction in the light of narrative mode-A case study of Robert van Gulik. Journal of Beijing International Studies University, (4), 43-49.

WANG, H. Y. (2016). Towards a universal theory of translation text-less back translation reviewed with examples from Judge Dee Mystery and Other Works. Shanghai Journal of Translators, (1), 1-9.

WEI, Q. (2006). Case-solving and detective novels: Starting from Dee Gong An. Journal of Yunnan University (Social Sciences), (4).

WEI, Y. (2009). Cross-cultural circulation of Judge Dee Stories. Comparative Literature in China, (1), 80-92.

XU, T. P. (2012). Dynamic equivalence theory and domestication in the Judge Dee Mysteries' Chinese version. Journal of Kaoshi Weekly, (52), 27-28.

ZHANG, P. (2010). Robert van Gulik: A diplomat between Sino-Western cultures. Beijing: Zhonghua Book Company.

ZHAO, Y. H. (Feb.13, 2002). The Dutch wrote Judge Dee. Chinese Readers’ Newspaper.

ZUO, M. Q., \& Hu, Q. (2015). van Gulik’s Judge Dee Mysteries' improvement and development from traditional Chinese Gong'an fiction. Journal of Chongqing Radio \& TV University, (3), 69-73. 